\title{
Physical Rehabilitation of a Spastic Diplegic Cerebral Palsy Patient - A Case Study
}

\author{
Namrata Rathi ${ }^{1}$, Pankhuri Multani ${ }^{2}$, Anagha Armarkar ${ }^{2}$, Amrita Sakharwade $^{3}$ \\ ${ }^{1}$ Professor, Department of Neurosciences Physiotherapy, Shri K. R. Pandav College of Physiotherapy, \\ Bhilewada, Bhandara, India. \\ ${ }^{2}$ Professor, Smt. Radhikatai Pandav College of Physiotherapy, Nandanvan, Nagpur, India. \\ ${ }^{3}$ Assistant Professor, Smt. Radhikatai Pandav College of Physiotherapy, Nandanvan, Nagpur, India. \\ Corresponding Author: Namrata Rathi
}

DOI: https://doi.org/10.52403/ijhsr.20220315

\begin{abstract}
Cerebral palsy $(\mathrm{CP})$ is a group of motor deficits induced by non-progressive brain damage in children. $\mathrm{CP}$ is a geographic ailment that affects both developed and developing countries with equal frequency. The study's purpose is to help Cerebral Palsy patients better their rehabilitation. The child's problems are being unable to stand without assistance due to truncal imbalance, having poor cognitive and social conduct, and being unable to do daily living activities independently. Children with CP usually have normal anatomic hip alignment when they are born. A variety of factors influence development, including delayed motor milestones and soft tissue anomalies, such as a muscle tone imbalance with strong hip flexors and adductors vs weaker hip extensors and abductors. When the GMFCS was used to evaluate the patient, the diagnosis of Spastic Diplegia cerebral palsy was confirmed. Physiotherapy intervention has been demonstrated to be useful in minimizing problems and improving patient outcomes.

Results: These studies revealed the difficulty of evaluating children with cerebral palsy. Clinical PTs must be aware of these complications and the fact that more than one assessment may be required to capture the children with CP's specific skills and behaviors.

Conclusion: Because of PT therapy, the patient was able to control his posture, regulate his head and neck, and stand with limited assistance despite wearing a KAFO.
\end{abstract}

Keywords: Cerebral palsy, Assessment, Spastic diplegia, Early intervention, Physiotherapy rehabilitation

\section{INTRODUCTION}

Cerebral palsy $(\mathrm{CP})$ is a diverse movement condition that affects kids born at all gestational stages but is more severe in preterm babies. As a result of nonprogressive anomalies in the growing fetus or infant's brain, CP refers to a collection of chronic mobility and postural developmental illnesses that induce activity restriction. This type of cerebral palsy affects both sides of the body, with the lower limbs gaining precedence. Spastic diplegia has been linked to more global developmental delays and moderates to severe intellectual disability in term-born children than in preterm-born children. The global incidence of cerebral palsy in the general population is estimated to be between 0.1 and 0.2 percent of all live births in developed countries, according to the data. (2). In poor countries, it is slightly higher, with the likelihood of increased $\mathrm{CP}$ with diminishing Gait also contributes to increases in muscle composition and alterations in motor function, resulting in an abnormal adaptation in body posture and an unstable gait. Ankle contractions are common in children with cerebral palsy, and 
they are most likely caused by structural alterations in the gastrocnemius and soleus muscles (3). The majority of people with $\mathrm{CP}$ contracts show that passive mutual motion is not present (4). The mechanisms that cause contracture are unknown. Physical activity benefits all children since it has been related to improvements in energy, stamina, self-esteem, social participation, and overall enjoyment. Specific spatial and temporal muscle activation may be absent in children with cerebral paralysis (CP) (5). Motor training and forced use throughout the early stages of development might cause physiological brain organization to be disrupted. On the other hand, children who were referred earlier improved their motor development more during the follow-up examination than those who were referred later. (6). Cerebral palsy (CP) affects youngsters and produces a wide range of symptoms and difficulties. The therapist must choose evaluations that appropriately represent the desired domain outcome metrics for the individual $\mathrm{CP}$ when conducting occupational therapy (OT) and physical therapy (PT) therapies (7).

\section{PATIENT INFORMATION}

The paper presents a case of a 5-year-old male kid with spastic diplegia who was diagnosed with using the GMFCS evaluation scale, I was diagnosed with stage $\mathrm{V}$ spastic diplegic cerebral palsy. The patient was born on time and without any unusual signs or symptoms, according to a family member. When he was two months old, he met with an accident. His family members first recognized that he was unable to crawl or execute supported standing when he was two years old, but they ignored the problem. At the age of five, the youngster was diagnosed with spastic diplegia, and physiotherapy was immediately begun.

CLINICAL FINDINGS: The youngster in this study showed deficits across the body, with a focus on the lower limbs and trunk. Gross motor and self-care functions were both harmed. The patient was completely reliant on the family member and would be unable to function without them. He could sit, but his truncal balance was severely damaged. The lower limbs showed symmetrical spasticity, tightness of the hip adductor and flexor muscles, and a scissoring gait pattern. The cardiovascular and respiratory systems were also normal. His social abilities had deteriorated as he grew older. GMFCS was used to further evaluate the youngster.

\section{PHYSIOTHERAPY INTERVENTION:}

A qualified neuro-physiotherapist provided physiotherapy to the youngster for one 1modailyasis in the Neuro-physiotherapy OPD. The purpose of the workouts was to improve her head and neck control. Swiss ball to and fro movements were used to supplement the training. The patient was given proper brush and ice techniques as well as prolonged stretching. The patient's physiotherapy sessions were planned to assess his functional balance by stimulating movement variations to establish his automatic correction and equilibrium reactions in a variety of situations. The patient did static weight-bearing activities daily. The child's body was subjected to challenge-specific moderate pressure to modify the application of force in different directions, allowing the individual to respond to a novel environment. The entire exercise regimen was designed to improve truncal balance and movement transitions while maintaining necessary safety precautions. The patient's caretaker was instructed on how to perform the home exercises according to protocol. He was fitted with a Knee Ankle Foot Orthosis (KAFO) to help keep her balance while standing, and he was able to stand with minimal assistance.

\section{RESULT}

His cognitive abilities improved faster than his gross motor coordination. After three months of physiotherapeutic operations, he was able to improve his head and neck control and balance her posture. 
He was able to stand with minimal support using a Knee Ankle Foot Orthosis (KAFO).

\section{DISCUSSION}

In industrialized countries, this is the most common clinical subtype of cerebral paralysis, while it is the second most common in developing countries. However, as indicated by a recent study found that the percentage of spastic diplegic cases increased from $22 \%$ to 34.5 percent over the last decade (8). Cerebral palsy (CP) is a term that refers to a group of developmental impairments that affect mobility and posture and create activity impairment. Hip dysplasia, which is caused by stiffness and rigidity of the hip adductor and hip flexor muscles, is the second most common musculoskeletal defect in cerebral palsy children (1).

It is only second to equines. Because children with GMFCS stages IV or V are at a higher risk of hip displacement, early use of posture control devices has been found to avoid hip difficulties. Standing services are recommended for children with cerebral palsy who are unable to stand by the age of 12-18 months and fall under the Postural Management System. Weight-bearing should be introduced into the standing regimen for CP-affected children aged 1218 months. In addition to a regular physical treatment routine, the standing technique involved the use of a frame for hip abduction. The standing group began the program at the age of $12-14$ months and completed it at the age of five years (3). At some point in their development, many children with high muscle tone (spastic CP) have limited passive range of motion and skeletal muscle abnormalities, which frequently worsen as they become older. The use of botulinum toxin, orthotics, and orthopedic procedures to treat restricted PROM has a significant impact on their mobility and quality of life. Although cerebral palsy is essentially a disease of gesture and posture, it also includes issues with vision and cognition, such as attention deficits and poor executive abilities, which need the utilization of several components of the cognitive memory region. (5).

\section{CONCLUSION}

According to the findings of the study, physiotherapy treatment such as weight-bearing exercises, the Roods approach, sensory integration, home exercise program, and the use of a Knee Ankle Foot Orthosis (KAFO) improved gross motor functions and social skills while having a minor impact on GMFCS.

Author's Contribution: All authors made the best contribution to the concept, assessment and evaluation, data acquisition, analysis, and interpretation of the data.

Informed Consent: Proper consent was taken from the patient for writing the case report.

Funding: This research received no external funding.

Conflict Of Interest: The authors declare no conflict of interest.

\section{Acknowledgement: None}

\section{REFERENCES}

1. Di Lieto MC, Brovedani P, Pecini C, Chilosi AM, Belmont V, Fabbro F, et al. Spastic diplegia in preterm-born children: Executive function impairment and neuroanatomical correlates. Res Dev Disabil. 2017 Feb;61:116-26.

2. D'Souza A, Bolsterlee B, Lancaster A, Herbert RD. Muscle architecture in children with cerebral palsy and ankle contractures: an investigation using diffusion tensor imaging. Clin Biomech. 2019 Aug;68:20511.

3. Jauhari P, Singhi P, Sankhyan N, Malhi P, Vyas S, Khandelwal N. A Comparison of Spastic Diplegia in Term and Preterm-Born Children. J Child Neurol. 2018 Apr;33(5): 333-9.

4. Garfinkle J, Li P, Boychuck Z, Bussières A, Majnemer A. Early Clinical Features of Cerebral Palsy in Children Without 
Perinatal Risk Factors: A Scoping Review. Pediatr Neurol. 2020 Jan;102:56-61.

5. Peters C, Chang A, Morales A, Barnes K, Allegretti A. An integrative review of assessments used in occupational therapy interventions for children with cerebral palsy. Cad Bras Ter Ocupacional. 2019;27(1):168-85.

6. Macias-Merlo L, Bagur-Calafat C, Girabent-Farrés M, A. Stuberg W. Effects of the standing program with hip abduction on hip acetabular development in children with spastic diplegia cerebral palsy. Disabil Rehabil. 2016 May 21;38(11):1075-81.

7. Spittle AJ, Morgan C, Olsen JE, Novak I, Cheong JLY. Early Diagnosis and
Treatment of Cerebral Palsy in Children with a History of Preterm Birth. Clin Perinatol. 2018 Sep;45(3):409-20.

8. Wright FV, Lam CY, Mistry B, Walker J. Evaluation of the Reliability of the Challenge when used to Measure Advanced Motor Skills of Children with Cerebral Palsy. Phys Occup Ther Pediatr. 2018 Aug 8;38(4):382-94.

How to cite this article: Rathi N, Multani P, Armarkar A et.al. Physical rehabilitation of a spastic diplegic cerebral palsy patient - a case study. Int J Health Sci Res. 2022; 12(3): 119-122. DOI: $\quad$ https://doi.org/10.52403/ijhsr.20220315 